\title{
Magnetic phase diagram of the semi-Heusler alloys from first principles
}

\author{
E. Şaşıŏlua) \\ Max-Planck-Institut für Mikrostrukturphysik, Weinberg 2, D-06120 Halle, Germany and Institut für \\ Festkörperforschung, Forschungszentrum Jülich, D-52425 Jülich, Germany \\ L. M. Sandratskii ${ }^{\text {b) }}$ and P. Bruno ${ }^{\text {c) }}$ \\ Max-Planck-Institut für Mikrostrukturphysik, Weinberg 2, D-06120 Halle, Germany
}

(Received 31 August 2006; accepted 19 October 2006; published online 30 November 2006)

\begin{abstract}
The magnetic phase diagram of the Mn-based semi-Heusler alloys is determined at $T=0$ using first-principles calculations in conjunction with the frozen-magnon approximation. The authors show that the magnetism in these systems strongly depends on the number of conduction electrons, their spin polarization, and the position of the unoccupied Mn $3 d$ states with respect to Fermi energy. Various magnetic phases are obtained depending on these characteristics. The conditions leading to diverse magnetic behavior are identified. The obtained results are in very good agreement with the available experimental data. (C) 2006 American Institute of Physics.
\end{abstract}

[DOI: $10.1063 / 1.2398892]$

Recent rapid development of spin electronics intensified the search on the ferromagnetic materials with tunable physical characteristics. The Heusler alloys form a particularly interesting class of systems. These systems have crystal structures and lattice parameters similar to many compound semiconductors, high Curie temperatures, and high spin polarization at the Fermi level. Some of them were found to have a half-metallic ground state, which is characterized by a $100 \%$ carrier spin polarization. ${ }^{1}$ These properties make the Heusler alloys particularly attractive for use in spintronics devices. Further prominent physical properties of this class of materials are the martensitic transformations. ${ }^{2}$ At low temperatures several Heusler compounds (i.e., $\mathrm{Ni}_{2} \mathrm{MnGa}$ and $\mathrm{Co}_{2} \mathrm{NbSn}$ ) undergo a structural transformation from a highly symmetric cubic austenitic phase to a low symmetry martensitic phase giving rise to two unique effects: magnetic shape memory effect and inverse magnetocaloric effect. Both features are regarded promising for the development of smart materials for future technological applications. An interesting combination of physical properties makes Heusler alloys the subject of intensive experimental and theoretical investigations. $^{3-10}$

To predict Heusler compounds with desired magnetic properties the theoretical understanding of the exchange interactions in these systems plays an important role. Our previous studies on experimentally well established Ni-based compounds $\mathrm{Ni}_{2} \mathrm{MnZ}(Z=\mathrm{Ga}, \mathrm{In}, \mathrm{Sn}, \mathrm{Sb})$ revealed a complex character of the magnetism in these systems. In particular, the obtained long range and oscillatory behavior of the exchange interactions as well as their strong dependence on the $s p$ atom (Z) gave an evidence for the conduction electron mediated exchange mechanism in Heusler alloys. ${ }^{11}$ The situation is not so different from the experimental point of view. Early measurements by Webster and Ziebeck on quaternary Heusler alloys $\mathrm{Pd}_{2} \mathrm{MnIn}_{1-x} \mathrm{Sn}_{x}$ and $\mathrm{Pd}_{2} \mathrm{MnSn}_{1-x} \mathrm{Sb}_{x}$ (Ref. 2) and recent studies on Mn-based semi-Heusler compounds $\mathrm{Ni}_{1-x} \mathrm{Cu}_{x} \mathrm{MnSb}$ and $\mathrm{AuMnSn}{ }_{1-x} \mathrm{Sb}_{x}$ demonstrated the impor-

\footnotetext{
${ }^{a)}$ Electronic mail: e.sasioglu@fz-juelich.de

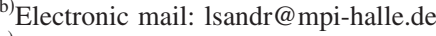

${ }^{c)}$ Electronic mail: bruno@mpi-halle.de
}

tance of the $s p$ electrons in establishing magnetic properties. ${ }^{12,13}$ In particular, the magnetic order and the Curie temperature in the latter compounds have been found to be quite sensitive to the $s p$-electron number.

The complexity of the exchange interactions complicates theoretical design of the systems with given magnetic characteristics and calls for further studies directed to find parameters of the systems governing magnetic properties and allowing their targeted change. Pursuing this aim we performed systematic calculations on semi-Heusler alloys CuMn $Z$ and PdMn $Z$ for different $Z$ constituents (In, Sn, Sb, Te, and their mixture treated in the virtual crystal approximation $^{14}$ ) focusing on the microscopical mechanisms of the formation of the long range magnetic order. We determine the magnetic phase diagram of these systems at zero temperature and estimate the $T_{C}$ of the compounds having ferromagnetic ground state within mean-field approximation. The calculational method is described elsewhere in detail. ${ }^{11}$ We use the lattice constants of 6.26 and $6.09 \AA$ for $\operatorname{PdMn} Z$ and $\mathrm{CuMnZ}$, respectively. ${ }^{2}$

We begin with the discussion of the exchange interactions calculated within the frozen-magnon approximation. ${ }^{15}$ Figure 1 presents the obtained exchange parameters between first four nearest neighbors of the $\mathrm{Mn}$ atoms in $\operatorname{PdMnZ}$ and $\mathrm{CuMn} Z$, as a function of the $s p$-electron number of the $Z$ constituent. The corresponding Curie temperatures are given in Fig. 2. In agreement with the experiments and the results of our previous calculations we obtain a strong dependence of the exchange parameters on the $Z$ constituent. As it is seen from Fig. 1 all exchange parameters oscillate between ferromagnetic and antiferromagnetic values with the variation of the $s p$-electron concentration. Considering first two nearest neighbor exchange parameters we see that they have ferromagnetic character for a broad range of compositions and dominate over the rest parameters playing a decisive role in determining the magnetic phase diagram (see Fig. 2). The absolute value of the parameters (we present only four nearest neighbor parameters) decays quickly with increasing interatomic distance. The parameters show the RudermanKittel-Kasuya-Yosida- (RKKY) type oscillations. 


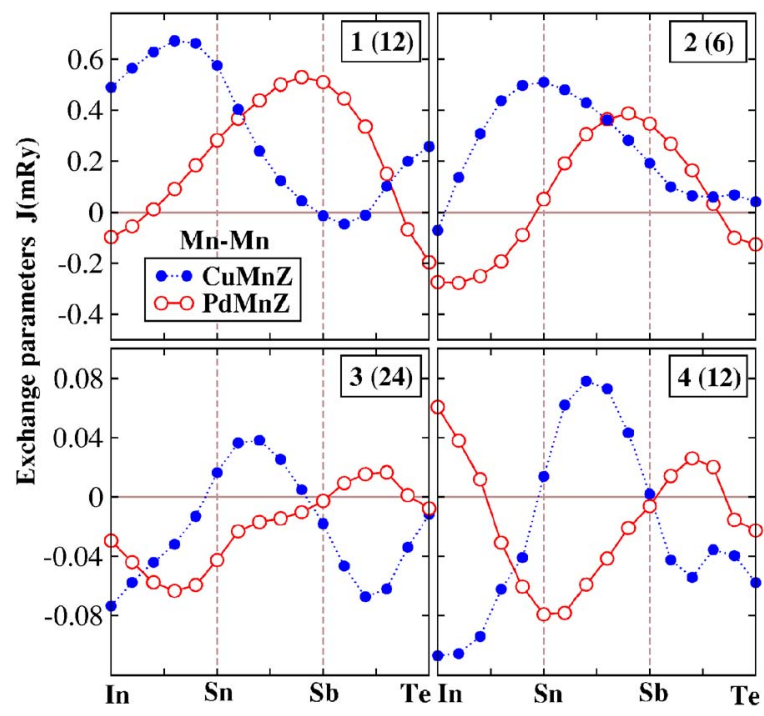

FIG. 1. (Color online) Exchange interactions between first four nearest neighbors of the $\mathrm{Mn}$ atoms in $\mathrm{PdMnZ}$ and $\mathrm{CuMnZ}$ as a function of the $s p$-electron number of the $Z$ constituent. Number of atoms within corresponding coordination spheres are also given.

This complex behavior of the exchange interactions appeared as a rich spectrum in the magnetic phase diagram (see Fig. 2), where magnetic order changes with $s p$ electron concentration. Furthermore, an interesting observation for the magnetism in these systems is that the maximum of the exchange interactions for both $\operatorname{PdMnZ}$ and $\mathrm{CuMnZ}$ corresponds to the similar number of the $s p$ electrons. The shift of the maxima for two systems is explained by the fact that $\mathrm{Pd}$ has one $s p$ electron less than $\mathrm{Cu}$. The properties of the exchange interactions are reflected in the properties of the $\mathrm{Cu}$ rie temperature (see Fig. 2) where we also obtained a relative shift of the maxima of the two curves corresponding to one sp electron.

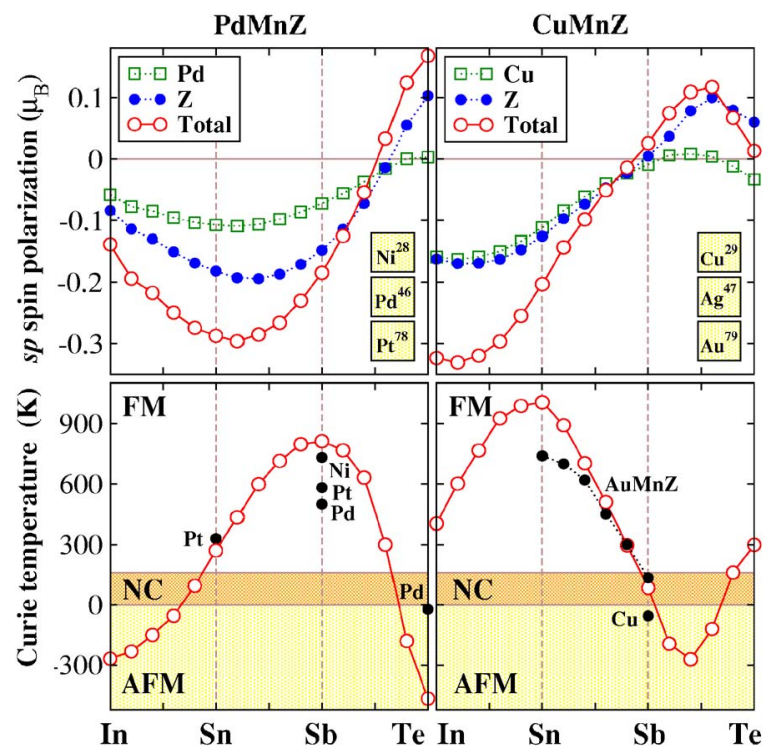

FIG. 2. (Color online) Upper panels: The $s p$-electron spin polarization of the $X=\mathrm{Pd}, \mathrm{Cu}$, and $Z$ constituents. Lower panels: Ground state magnetic phase diagram and mean-field Curie temperature $\left(T_{C}^{\mathrm{MFA}} \sim J_{0} \equiv \Sigma_{R \neq 0} J_{0 R}\right)$ of PdMnZ and CuMnZ. FM, NC, and AFM stand for ferromagnetic, noncollinear, and antiferromagnetic orderings, respectively. Experimental values of the Curie temperatures (filled spheres) are taken from Refs. 2 and 13.
Combination of these features with the large distance between $\mathrm{Mn}$ atoms $\left(d_{\mathrm{Mn}-\mathrm{Mn}}>4 \AA\right)$ makes the assumption natural that the exchange coupling mechanism in Heusler alloys is indirect and takes place via the conduction electrons. A RKKY-type coupling seems to be the most probable one; however, the rich spectrum of magnetic orderings obtained by varying $s p$-electron concentration (Fig. 2) makes the interpretation of the results in terms of existing theories difficult and calls for a detailed analysis. This is explained by the fact that the density functional theory (DFT) is not based on a model Hamiltonian approach and does not use a perturbative treatment. Thus, various exchange mechanisms appear in the calculational results in a mixed form and a separation of them into individual contributions is not easy. In this case the model Hamiltonian studies relevant to the problem provide useful information for a qualitative interpretation of the DFT results. Among such approaches the Anderson $s$ - $d$ model ${ }^{16}$ is the most suitable for Heusler alloys because of the localized nature of the magnetism in these systems. ${ }^{2}$ Within this model the effective exchange interaction between distant magnetic atoms can be separated into two contributions: $J_{\text {indirect }}=J_{\mathrm{RKKY}}+J_{S} \cdot{ }^{17}$ The first term is a RKKY-like ferromagnetic term which stems from the interaction between the local moment and the conduction electron states inducing a spin polarization in conduction electron sea. In general, this interaction contains two distinct processes: electrostatic Coulomb exchange interaction and $s p-d$ mixing interaction. The former induces a net positive spin polarization while the contribution of latter is always negative and disappears in strong magnetic limit. ${ }^{18}$ Note that the amplitude of this polarization determines the strength of the RKKY-like exchange coupling. However, the second term has different origin; it arises from virtual-charge excitations in which electrons from local $d$ states of the Mn are promoted above the Fermi sea providing an additional contribution to the indirect exchange coupling. This term depends mostly on the distance of the unoccupied Mn $3 d$ peaks from the Fermi level. The closer the peaks to the Fermi level the stronger the $J_{S}$. Moreover, in contrast to the first term, this second term is always antiferromagnetic and its strength decays exponentially with distance. The overall magnetic behavior depends on competition of these two terms as exemplified by the present systems.

Now we turn to the interpretation of the DFT results in terms of these two mechanisms. To estimate the relative contribution of the ferromagnetic RKKY-like exchange we present in Fig. 2 the calculated conduction electron spin polarization in $\operatorname{PdMn} Z$ and $\mathrm{CuMn} Z$ as a function of the electron number of the $Z$ constituent. The analysis of the calculational data allows to make a number of important conclusions. First, in both systems the direction of the induced spin polarization is opposite to the direction of the Mn moment in a broad interval of compositions. This feature reveals the primary role of underlying $s p-d$ mixing interaction in exchange coupling and justifies the use of the Anderson $s$ - $d$ model for the description of the magnetism in these systems. It is worth to note that, in agreement with the present findings, recent experiments on ferromagnetic full-Heusler compounds $\mathrm{Cu}_{2} \mathrm{MnAl}$ and $\mathrm{Ni}_{2} \mathrm{MnSn}$ gave a large conduction electron spin polarization that is antiferromagnetically coupled to $\mathrm{Mn}$ magnetic moment. ${ }^{19,20}$

Another remarkable feature we obtained is a very clear relation between the spin polarization and the mean-field $\mathrm{Cu}-$
AIP license or copyright, see http://apl.aip.org/apl/copyright.jsp 


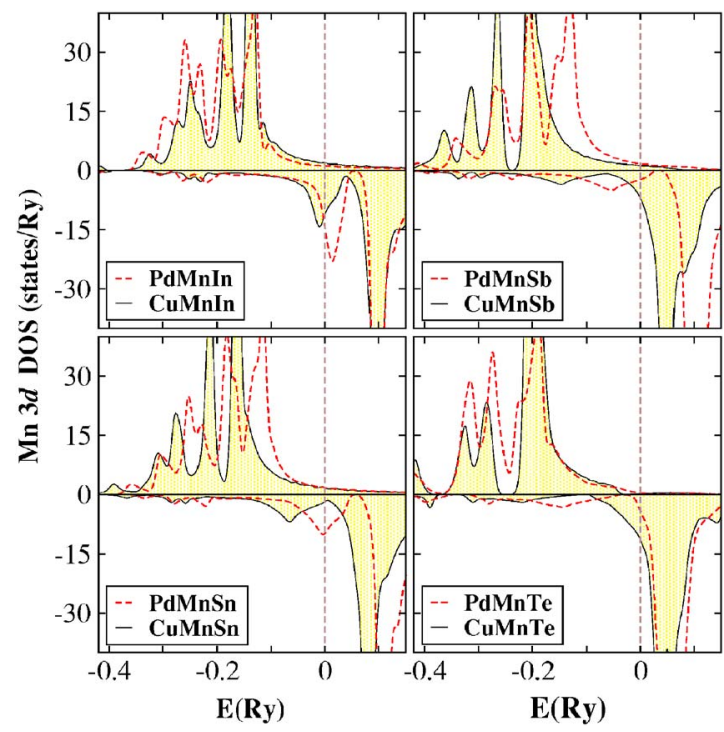

FIG. 3. (Color online) Spin resolved Mn $3 d$ density of states in PdMnZ and $\mathrm{CuMnZ}(Z=\mathrm{In}, \mathrm{Sn}, \mathrm{Sb}, \mathrm{Te})$ for stoichiometric compositions. Vertical broken lines denote the Fermi level.

rie temperature (or exchange parameters) in a large part of the phase diagram (see Fig. 2). Indeed, the compounds with very large spin polarization are characterized by very high Curie temperatures. Interestingly, for the zero polarization also the Curie temperature vanishes reflecting the dominating character of the ferromagnetic RKKY-like exchange mechanism in establishing magnetic properties. However, at some regions superexchange mechanism becomes important. This can be seen in Fig. 2 where for $\mathrm{PdMnIn}_{1-x} \mathrm{Sn}_{x}(x<0.8)$ system we obtain an antiferromagnetic order in spite of very large spin polarization. To gain further insight into the nature of this coupling we present in Fig. 3 the spin resolved Mn $3 d$ density of states of PdMnZ and CuMnZ for stoichiometric compositions. As pointed out earlier this mechanism is more sensitive to the properties of the unoccupied Mn $3 d$ peaks. For PdMnIn there is a large peak just above the Fermi level and as a result the antiferromagnetic superexchange dominates over the ferromagnetic RKKY exchange giving rise to an antiferromagnetic (AFM) order. In transition from In to Sn this peak gradually decreases and, therefore, the superexchange becomes less important. Around $\mathrm{Z}=\mathrm{Sb}$ this peak almost disappears leading to the FM order. However, another large peak becomes close to the Fermi level when $Z$ approaches Te. This turns the system from a ferromagnetic state into an antiferromagnetic one with a non-collinear ordering in between due to competition of these two mechanisms. The situation is very similar in the case of $\mathrm{CuMnZ}$ where we also obtain a rich magnetic behavior.

Finally, we would like to comment on the magnetic behavior of the systems having non-magnetic $X$ or $Z$ elements from the same column of the Periodic Table $(X=\mathrm{Ni}, \mathrm{Pd}, \mathrm{Pt}$, or $\mathrm{Cu}, \mathrm{Ag}, \mathrm{Au}, Z=\mathrm{Al}, \mathrm{Ga}, \mathrm{In}$, etc.). For these nonstudied compounds we expect a similar behavior on the basis of previous experimental and theoretical studies. ${ }^{2,11}$ Indeed, as seen in Fig. 2 the $X \mathrm{MnSb}$ compounds with $X=\mathrm{Ni}, \mathrm{Pd}, \mathrm{Pt}$ have similar values of the Curie temperature and are strongly ferromagnetic. Our results for ground state magnetic order and finite temperature properties compare well with the existing experimental data. In particular, the observed trend in the variation of the Curie temperature of $A u M n S n n_{1-x} \mathrm{Sb}_{x}$ is very well described by our calculations. ${ }^{13}$

In conclusion, the magnetic behavior of the semiHeusler alloys can be described in terms of the competition of two exchange mechanisms: ferromagnetic RKKY-like exchange and antiferromagnetic superexchange. We found that each mechanism depends on certain parameter. In the case of large conduction electron spin polarization and the unoccupied Mn $3 d$ states lying far from the Fermi level, a RKKYlike ferromagnetic interaction is dominating while antiferromagnetic superexchange becomes important in the presence of the peaks of unoccupied Mn $3 d$ states close to the Fermi energy. Various magnetic phases are obtained depending on these characteristics. These findings suggest a practical tool for the design of the materials with given properties.

${ }^{1}$ R. A. de Groot, F. M. Mueller, P. G. van Engen, and K. H. J. Buschow, Phys. Rev. Lett. 50, 2024 (1983).

${ }^{2}$ P. J. Webster and K. R. A. Ziebeck, in Alloys and Compounds of d-Elements with Main Group Elements, New Series, Group III, Vol. 19/c, Pt 2., edited by H. R. J. Wijn (Springer, Berlin, 1988).

${ }^{3}$ I. Galanakis, P. H. Dederichs, and N. Papanikolaou, Phys. Rev. B 66, 134428 (2002).

${ }^{4}$ I. Galanakis, M. Ležaić, G. Bihlmayer, and S. Blügel, Phys. Rev. B 71, 214431 (2005).

${ }^{5}$ J. Rusz, L. Bergqvist, J. Kudrnovský, and I. Turek, Phys. Rev. B 73, 214412 (2006).

${ }^{6}$ Y. Kurtulus, R. Dronskowski, G. D. Samolyuk, and V. P. Antropov, Phys. Rev. B 71, 014425 (2005).

${ }^{7}$ S. Picozzi, A. Continenza, and A. J. Freeman, Phys. Rev. B 66, 094421 (2002).

${ }^{8}$ T. Zayak, P. Entel, J. Enkovaara, A. Ayuela, and R. M. Nieminen, Phys. Rev. B 68, 132402 (2003).

${ }^{9}$ I. Takeuchi, O. O. Famodu, J. C. Read, M. A. Aronova, K.-S. Chang, C. Craciunescu, S. E. Lofland, M. Wuttig, F. C. Wellstood, L. Knauss, and A. Orozco, Nat. Mater. 2, 180 (2003).

${ }^{10}$ T. Krenke, E. Duman, M. Acet, E. F. Wassermann, X. Moya, L. Mañosa and A. Planes, Nat. Mater. 4, 450 (2005).

${ }^{11}$ E. Şaşıoğlu, L. M. Sandratskii, and P. Bruno, Phys. Rev. B 70, 024427 (2004).

${ }^{12}$ S. K. Ren, W. Q. Zou, J. Gao, X. L. Jiang, F. M. Zhang, and Y. W. Du, J. Magn. Magn. Mater. 288, 276 (2005).

${ }^{13}$ C. Walle, L. Offernes, and A. Kjekshus, J. Alloys Compd. 349, 105 (2003).

${ }^{14}$ J. M. Schoen, Phys. Rev. 184, 858 (1969).

${ }^{15}$ S. V. Halilov, H. Eschrig, A. Y. Perlov, and P. M. Oppeneer, Phys. Rev. B 58, 293 (1998).

${ }^{16}$ P. W. Anderson, Phys. Rev. 124, 41 (1961).

${ }^{17}$ Z.-P. Shi, P. M. Levy, and J. L. Fry, Phys. Rev. B 49, 15159 (1994).

${ }^{18}$ R. E. Watson, A. J. Freeman, and S. Koide, Phys. Rev. 186, 625 (1969).

${ }^{19}$ E. Zukowski, A. Andrejczuk, L. Dobrzyñski, M. J. Cooper, A. G. Dixon, S. Gardelis, P. K. Lawson, T. Buslaps, S. Kaprzyk, K.-U. Neumann, and K. R. A. Ziebeck, J. Phys.: Condens. Matter 9, 10993 (1997).

${ }^{20}$ A. Deb, N. Hiraoka, M. Itou, Y. Sakurai, M. Onodera, and N. Sakai, Phys. Rev. B 63, 205115 (2001). 